\title{
Téoros
}

Revue de recherche en tourisme

\section{Le trekking par les textes, ce qu'exprime le discours promotionnel}

\section{Jean Michaud}

Volume 13, numéro 3, automne 1994

Le tourisme d'aventure : vers la maturité ?

URI : https://id.erudit.org/iderudit/1077111ar

DOI : https://doi.org/10.7202/1077111ar

Aller au sommaire du numéro

Éditeur(s)

Université du Québec à Montréal

ISSN

0712-8657 (imprimé)

1923-2705 (numérique)

Découvrir la revue

Citer cet article

Michaud, J. (1994). Le trekking par les textes, ce qu'exprime le discours

promotionnel. Téoros, 13(3), 33-37. https://doi.org/10.7202/1077111ar d'utilisation que vous pouvez consulter en ligne.

https://apropos.erudit.org/fr/usagers/politique-dutilisation/ 


\title{
Le trekking par les textes, ce qu'exprime le discours promotionnel/(1)
}

\author{
Jean Michaud"
}

Le trekking est à la mode. Il ne sevend plus aujourd'hui un seul guide touristique qui, présentant une région du monde considérée exotique pour la clientèle d'ici et dotée d'un écosystème particulier, ne propose d'y effectuer un trekking. Mais qu'estce que trekker veut dire? Ne s'agit-il que d'une forme de l'activité physique, ou y germe-t-il une variété de discours sur FAutre, sur la rencontre? Que dit-on et que ne dit-on pas dans le discours promotionnel, et pourquoi? Sous les épithètes accumulés, la promotion active du trekking répond d'abord aux appétits de profitabilité des agences devoyages locales et transnationales, mais aussi aux besoins de particularisation des voyageurs et à leur désir profond de mériter les émotions vécues.

Que veut dire le verbe trekker? Pourquoi l'industrie de l'aventure en a-t-elle fait une activité privilégiée; comment en fait-elle la promotion? Et comment se fait-il qu'un concept aux frontières si floues, comme on le verra, puisse engendrer un intérêt si grand dans l'industrie touristique qu'il suffise à lui seul à déplacer en bloc yuppies, trippeux et secrétaires médicales jusqu'aux antipodes - de préference jusqu'aux antipodes - dans le but avoue de s'y livrer, ne serait-ce qu'une fois dans leur vie, à un exercice physiquement pénible, moralementéprouvant, et souventruineux. Un geste que toutel'organisation d'une vie par ailleurs confortable, fondée sur le travail, l'économie et la stabilité, semble contredire.

Au Québec, comme ailleurs dans le monde industrialisé, la promotion faite par les agences de voyages dites d'aventure ajoutée aux conseils d'amis voyageurs, aux articles de revues spécialisées comme Géo ou Grands reportages, aux excitantes soirées Grands explorateưrs, et à un bon nombre de guides touristiques spécialisés, ont tous pour but, avéré ou secret, d'éveiller l'aventurier qui sommeille en nous. Supposant

Monsieur Jean Michaud est anthropologue chercheur pour le Groupe d'etudes et de rectherches sur l'Asie contemporaine (GERAC), a la Faculté des sciences sociales de I'Université Laval.

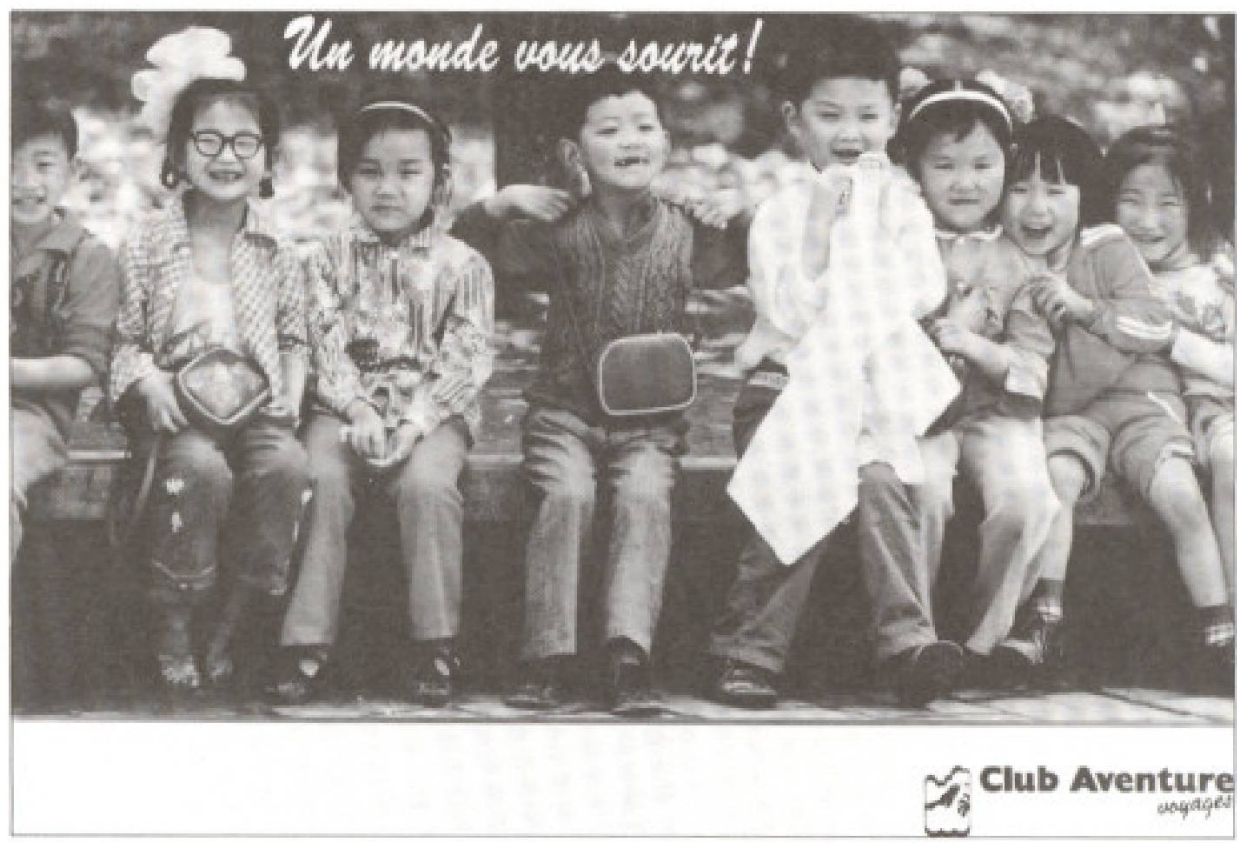

que cet éveil se produise, l'intrépide, s'il est conséquent, doit passer à la recherche active d'un moyen de donner une forme à cette aventure. Ces mêmes sources d'information, jugées par un grand nombre comme hautement crédibles, ont encore une fois la réponse recherchée. Entre toutes, la méthode privilégiće pour vaincre le Sauvage, aussi bien que pour devenir son ami, se nomme le trekking. Aucune étude à ma connaissance n'a encore été consacréc à ce sujet, et il est intéressant de tenter de cerner leconceptet de vérifier les assises de sa pratique.

\section{Tenter de définir le trekking, une aventure en soi}

Pour définir le trekking, un renvoi aux dictionnaires est une première étape utile. A la rubrique trek, le Collins indique: $* 1 . A$ long and often difficult journey. 2. South African. A journey or stage of a journey, especially a migration by ox wagon». Plus defini, mais moins savant, le Petit Robert proposeà trekking: (1975, anglais): Randonnée touristique dans des régions difficilement accessibles $\%$. Il va sans dire que si l'origine du terme semble effectivement liée aux migrations internes des colons afrikaners au $\mathrm{XIX}^{*}$ siècle au moment d'établir des fronts pionniers dans l'intérieur sud-africain, des acceptions modernes font du trekking une activité davantage ancrée dans la réalité du jour. Si les concepteurs du célèbre Star Trek, transposition surréaliste du go $W_{e s t}$ américain, ont bien gardé le sens de migration colonisatrice, il reste effectivement peu de gens pour unir le terme au déplacement des populations et au char à boeufs. Ce pourrait être l'industrie touristique qui aurait ravivé le mot dans les années 1970 , lorsque sont devenus de plus en plus populaires les voyages d'agrément promouvant de se déplacer à pied pour visiter un lieu ou une population. Pour des raisons obscures qui tiennent probablement au phénomène des modes et procèdent sans doute de la tactique de marketing prônant qu'il vaut mieux nommer les nouvelles activités avec des termes nowverux, cette mise au goût du jour commerciale est sans doute responsable de la connotation touristique que porte maintenant le mot dans le Petit Robert, et dans presque toutes les langues où on lui trouve un usage.

Dans l'édition touristique, loin des références étymologiques, une certaine préhension de la valeur concrète du con- 
cept est possible, mais a contrario. Précisons d'emblée que sur la quarantaine de guides touristiques faisant mention du trekking consultés ces dernières années, aucun ne propose de définir ce qu'est cette activité. A une exception près. La maison australienne Lonely Planet, la référence touristique la plus universellement parcourue parmi les guides écrits touchant au trekking, a mis sur le marché en 1979 et réédité tous les deux ans depuis, un ouvrage intitule Trekking in the Himalayas (Armington, 1982). Peut-être à cause de la nouveauté relative du terme dans le discours touristique de la fin des années 1970 , l'auteur a jugé bon de donner quelques précisions sur ce qu'est le trekking. Une trentaine de pages, citées ici à partir de l'édition 1982, abordent le sujet et \$a pratique sous des rubriques varióes. Bien que ces pages aient été spécifiquement écrites en pensant aux zones montagneuses du Népal, leur propos déborde largement ce adre géographique et il permet de jeter une lumiere utile sur le sens que peut revêtir le concept. Lorsque l'auteur commente la question : Pourquoi faire un trekking au Népal ?, il fournit en bloc une somme appréciable d'informations:

\section{[...] the Kingdom [of Nepal] must be visited in the slowest and most intimate manner - by walkings. [...] Each step provides new and intriguing viewpoints [...]. For the romanticist, Eacls step is a step in the footsteps of Hillary, Tenzing and otber Himalayn explorers. [...] You will encounter people in remote mountain villages whose life-style bas not cbanged in generations. [.. ] Atrek alsoprovides a glimpse into the ancient culture of Tibet [wbich] is now rapilly being altered in Nepal ly the influcnce of tourism and fortign-aid progran= mess. [...] Your preconceptions of the Himalloya alter as you turn from peaks faned only for their bigbt to gaze at far more picturesigue sunnits you bave never before beard of. (Amington, 1982:9- 10 , emplatse ajoute?}

Symptôme peut-être d'une difficulté à penser le trekking, il a semblé judicieux à "auteur de le définir en s"attardant précisément à tout ce qu'il n est pas. Par exemple, des rubriques annoncent $\alpha \mathrm{A}$ trek is not a wilderness experiences, ou $x$ A trek is not a climbing trip». On semble chercher d'emblée à corriger une compréhension qu'on considère possiblement erronée, suggérant l'hypothèse d'une opinion déjà faite

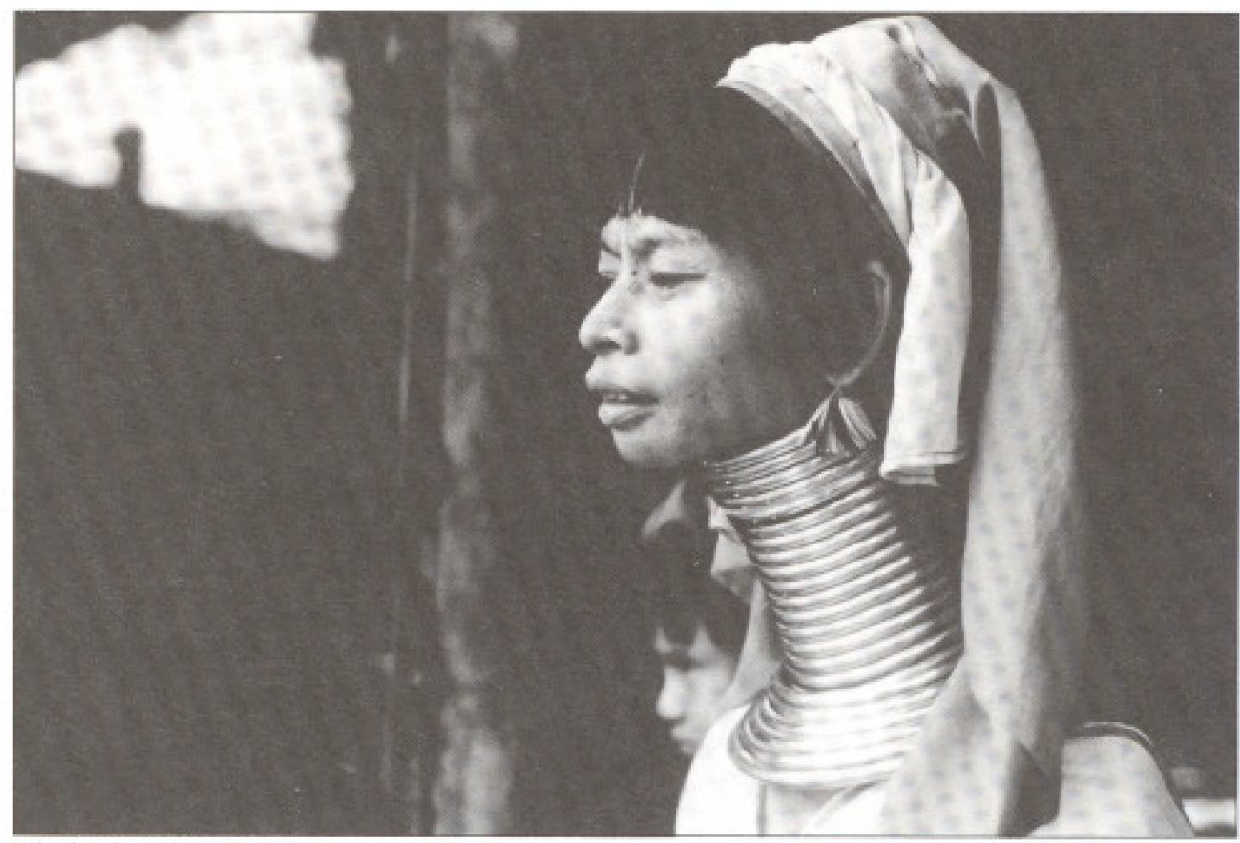

Thaillande, Club Aventure.

chez le lecteur, d'une prescience d'un ou plusieurs concepts apparentés, et qu'il faudrait rectifier. Le trekking pourrait être proche cousin de quelque chose d'autre avec lequel il serait aisément - et inopportunément - confondu. S'il ne faut pas s'attendre à pénétrer un écosystème à l'état sauvage, c'est que ait is impossible to get completely away from people [...] though isolation is traditionally a crucial element of any wilderness experiences (p. 10). Si le trekking n'est pas de l'alpinisme, c'est qu'au Népal, wthe trek is always on well-developed trails through villages and across mountain passes (p. 11). Suivant une logique révélatrice, cest également sous la rubrique $\ldots[. .$.$] not a climbing$ experiences qu'on déclare que l'essentiel de la beauté de l'Himalaya népali, c'est l'environnement, le cadre (surroundings), incluant: ofriendlypoople, picturecruevillages and a great variety of cultures and traditions thatscem to exemplify manyof the attributes we bave last in our headlong rash for development and progeres in the Westo ( $\mathrm{p}$. 12 , emphase ajoutée).

A la rubrique: $\propto$ Qui devrait faire un trekking? (p. 12), il $y$ a d'abord deux conditions préalables marquant clairement les deux faces du trekking touristique: aimer le grand air (dimension physique liée à la topographie), et powvor se sentir confortable parmi des societs differentes (dimension huinaine liée à la rencontre). On précise :

Anyone who bas walked extensively in the wountains bas all the shills neaesary for an extended trek in

\begin{abstract}
Nepal. [... If you insist on dragging your world around in your suitcase you will be miserable. [...] Trek with there thought in wind and you will enjoy the most rewarding experience of your life and develop new understanding of yourself and your world. (Armington 1982: 11, 13, 14, emphase ajoutée).
\end{abstract}

En somme, en faisant un trekking, l'on peut comprendreque Lonely Planet propose de briser la solitude de l'existence moderne, fruit amer de l'oubli des vérités d'antan, et ce au moyen de la rencontre intimeduvoyageur dépouillé avec un Autre qui, lui, est demeuré intact. Il s'agit ainsi de provoquer un tourbillon d'émotions porteur d'insights - pour employer un langage analytique qui $n^{7}$ est pas hors de propos ici - sur ce que nous sommes et ce qu'est le monde, une expếrience singulière gage d'entendement de l'état d'altérité. Rien de moins. Cette vision percutante est jugée suffisamment adéquate pour qu'on la réimprime depuis 14 ans, et elle n'a pas trouvé de contradicteurs. Elle marque le monde du tourisme d'aventure, et on la retrouve jusque dans le discours promotionnel du trekking touristique tel qu'on le fabrique au Québec.

\section{Le trekking tel que promu au Québec}

Un poids lourd dans le petit marché québécois, le Club Aventure est chez-nous 


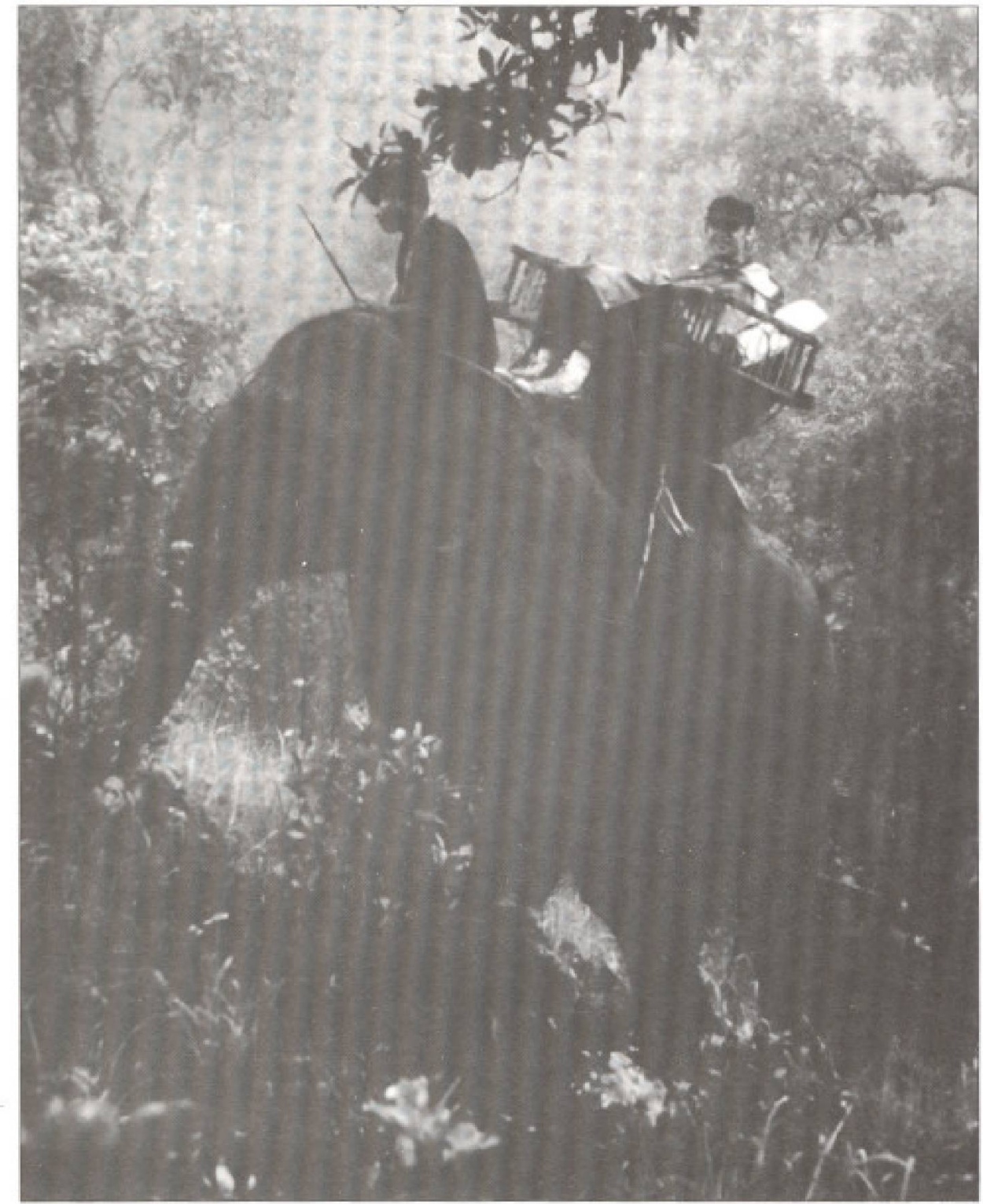

Thailande, Club Aventure.

l'agence numéro 1 de... l'aventure - borrdes sentien battus, précise-t-on. Si l'on en juge par le contenu de ses brochures promotionnelles, le Club Aventure, consciemment ou non, souscrit à l'ambiance décrite ci-haut tout en proposant une systématisation personnalisée du concept. Dans un imposant catalogue couleur de 1987 comptant 135 pages, un symbole pictural accompagné d'un chiffre est associé aux itinéraires comportant une activité physique notable. On le définit comme suit: $*$ Marche en montagne prévue. Randonnée de longue durée exigeant une certaine forme physiques (Club Aventure, 1987: 7). Graduant les propositions d'itinéraires en 4 niveaux de difficulté physique, le mot trekking n'apparaît que pour les deux niveaux les plus élevés. Il s'acoom- lenvironnement et d'awitié entre les peuples (ClubAventure, 1991:4). Dans ce discours nouvelle vague, un bémol appréciable est désormais accolé à la dimension physique du trekking au profit d'une emphase appuyant résolument la dimension humaine de l'expérience. En passant, on précise: *Nos guides peuvent conduire [...] des plongeurs vers la grande barrière de corail en Australie, des chasseurs de papillons dans la jungledu Brésil [... et] des ethnologues chez les tribus Bochimans du Botswanaw (Club Aventure, 1987: fiche signalétique, p. 3).

Unenuancedans le discours promotionnel au Québec mérite d'êtrerelevée. Ala façon du Club Aventure cuvée 1987, l'agence Expéditions Monde (Expéditions Monde, 1992), considerablement plus petite mais en activité depuis plus de 10 ans, utilise également des pictogrammes dans sa brochure 1992-1993 pour marquer les principales attractions des itinéraires offerts. Un seul mot est ici associé à chaque image, et on a choisi de distinguer les concepts de trek et de trilhu au moyen d'images differentes. Cependant, dans la plupart des itunéraires hors des pays fortement industrialisésoùl'un ou l'autresymbole apparaît, on les retrouve côte à côte, atténuant par le fait même la portée explicative de la distinction initiale. Ainsi au Québec, si l'on se fieau discours promotionnel dans le monde de l'aventure et de l'expédition, une certaine distinction entre faire un trekking et visiter des sociétés exotiques peut se penser, mais la tendance essentielle est de fondre en une seule activité les deux dimensions de l'effort physique et de l'expérience humaine. Ala manière Londy Planet.

\section{Un discours exemplaire : trekker en Thaillande}

pagne de termes comme: expédition, b1vouac, difficulté, haute altitude, parfaite condition physique. Dans cette brochure de 1987,8 destinations sur un total de 31 présentent le symbole de l'effort physique, dont 6 sont aux niveaux de difficulté supérieurs. Quatte ans plus tard, en 1991, dans unebrochurede formatplusmodeste (Club Aventure, 1991), lesconcepteursabandonnent niveaux dedifficultéet symboles, et ne gardent que 4 destinations où apparait le mot trekking dans le texte d'acoompagnement (ce sont le Sikim, le Népal, les Philippines et la Thaillande). Les anciens récipiendaires du symbole offrent maintenant ce qu'il est convenu d'appeler la randonnée pédestre. Celle-ci s'agrémente souvent d'une nouveauté, la découverte etbnologigue dans un contexte de ropect de
Un cas particulier où s'effectue le trekking touristique, peut être pris afin d'y voir ce qu'en fait le discours de la promotion et des guides. Peu de sources seront citées puisque le discours dominant est aisément cernable et quela répétition est importante d'un auteur à l'autre. Baffie (1989) a d'ailleursjudicieusement remarqué queles auteurs de guides touristiques semblent ne lire essentiellement que d'autres guides touristiques pour donner forme à leur propos.

La Thailande est certainement un hautlieu du trekking si l'on en juge par le nombre impressionnant d'agences de voyages proposant de trekker dans tel village primitif, isolé et intouché, ou si l'on 
fait la somme des volumes que lui consacre l'édition touristique internationale. Un ou deux auteurs en sciences sociales déclarent également étudier le phénomène du trekking dans les tribur montagnardes au norddupays, dont le prolifique Erik Cohen (1989 par exemple). Mais aucun n'a jugé bon de définir le concept étudié.

On relève plus d'une soixantaine de parutions touristiquesnotables sur la Thailande, et presque toutes comprennent une section sur le trekking dans le nord du pays. Je propose de voir quelques exemples parmi les plus utiles pour saisi l'étendue de l'éventail des propositions. Le trekker's Guide to the Hill Tribes of Northern Thailand (Davies \& Wu, 1990), publié en Thailandemême, propose une synthèse de l'activité :

\begin{abstract}
[Trekkingtours]generally take from four to six days. Tbey may visit quite remote areas, so you may see villages tbat bave not been exposed to westerners for very long. these trips can be nuch the most autbentic way of experiencing the life of tbe bill tribes. But be prepared for some discom fort and effort (pp. 1-2, emplase ajoutée).
\end{abstract}

En plus de bien annoncer dès le titre qu'il s'agira de trekker non seulement dans les montagnes, maiségalement chez les tribus montagnardes - le terme billtribe associe deux idées se complétant utilement - ce guide en vogue sur les lieux memes annonce clairement l'authentique ${ }^{i t}$ et le difficile, le but et la nécessaire façon de l'atteindre. J'ai retenu cette citation entre autre parce qu'elle s'emboite bien dans la typologie des motivations touristiques de Valene Smith (1989). Smith propose entre autres deux catégories de touristes qui, mises ensemble, décrivent adéquatement letrekkewren Thailande. Il s'agit du towrite etbwique (etbric tourism) et du towriste entromentental(enimomiental tourism). On le dit ethnique parce que l'adepte recherche chez les visités des particularités sociologiques et culturelles exotiques qui les distinguent et leur conferent un caractère uniguze et environnemental parce que l'écosystème visité ressort franchement du commun et, en plus d'étre une attraction en soi, permet des activités physiques exigeantes auxquelles tous ne peuvent prétendre. J "ajouterais de ma propre observation quele touristeethniqueetenvironnemental est généralement jeune et énergique, relativement sérieux, et mieux informé sur le pays visité que le touriste d'un genre plus récréatif. Il fuit les autres touristes, qu'il juge généralement avec sévérité, il réprouve la facilité, et il est conséquemment désireux de se mériter par un certain effort ce qu'il rapportera de souvenirs et d'émotions.

Revenons aux guides. Pour le monde francophone, Le guide du routard est publié chez Hachette comme un penchant brancbé de la prestigieuse collection Guides bleus. Immensément populaire auprès de la clientèle française et francophile de tous âges - on parled'un tirage de plus de 40000 exemplaires pour le seul numéro sur la Thailande-, il sied particulièrement à ceux qui sont désireux de woyager à très peu de frais, une vertu selon les auteurs. Ici, le trekking en Thailande se centre carrément sur la visite tribale, passant presque sous silence l'aspect physique de l'excursion. Sous la rubrique Treks dans les tribus montagrhorles, didactistne oblige, on prendd'abord soin d'informer le lecteur sur les tribar en question. Au sujet des Akha, par exemple, on prévient que:

\section{I... les Akba]se lavent très rarement (ils se néfient de l'cau). [...] $D_{E}$. tradition patriarcale et monogame, rien ne leur interdit véritablement d'avoir phusiewrs fommes et ils ne se gênent pas. Ca fait plas de main- d'ownre dans les champs et plus de temps libres pour l'bomme. Aucune fomme n'a encore eu lidée de se révolter. [...] (Le guide du routard, 1992: 119).}

Du même souffle, on ajoute: * La femme porte la minijupe ainsi que des jambières décorées qui leur [sic] donne une allure très sexys $(1992,119)$. Les Yao, quant à eux, étonnent:

\section{Le mariage procede d'un curiest rituel: le jewne bonmte doit choisir sa fenme à Pextérieur de son clan. Le pire de sa furure femme hi dewandera une somme astrono- migue, bo tout avec le sourire. Ensuite il emmènera sa prowise vive dbez ses parents [...] avec ha belle-meresur le dos toute ha journée! \\ (Le guide du routard, 1992:119).}

Les auteurs donnent à la rubrique Sawoirvive dans les tribus leurs conseils cardinaux pour éviter les malentendus durant le trekking, conseils qui sont au nombre de trois: We restez jamais assis devant une porte d'entrée [...] ne donnez pas de bonbons, cigarettes et gadgets [...] ne donnez jamais d'argent pour prendre une photow (p. 120). Le guide du routard est certai- nement l'un des cas les plus extrêmes de simplification outrancière, et le propos sur le trekking y est réduit à sa plus simple expression: un discours sur le Primitif comme spectacle, un Autre inassimilable a Soi, objet de curiosité et de dérision.

Plus romantique, le Club Aventure décrivait comme suit son itinéraire de 1987 dans le nord du pays:
Entreprendre 9 jours de trekking à
la rencontre de Karens et des Méos.
Vivre la sensation grisante de l'effort physique, contempler des paysage splendides, s'imprégner depaix, se fondre dans l inmensité. Rencontrer le cbef d'un de ces villages construits sur pilotis. Cbanter, discuter toute la nuit. (1987;41)

En donnant la priorité à la rencontre humaine, on retrouve très forte ici l'idée de l'expérience émotionnelle, humaniste, l'idée du retour sur sa propre intériorité concurremment à la fusion symbiotique avec l'immensité, avec l'Autre. Détail intéressant: «chanter, discuter toute la nuit» laisse à entendre que la communication est possible et que le temps compte pour peu, alors que les montagnards ne parlent ni ne comprennent d'autres langues que leurs langues vernaculaires ou le thai, et qu'ils doivent être levés avant l'aube pour être prêts à partir aux champs au lever du jour. La communication dont il est réellement question ici, c'est d'abord celle qui se fait avec les guides - le plus souvent des nonmontagnards-onentre dubstes, cequin'est que concordant avec la formule de l'itinérance grégaire du Club Aventure.

\section{Le Thailand. A Travel Survival Kit} (Cummings, 1990), publié chez l'incontournable Lonely Planet, offre dans l'ensemble une vision plutôt pondérée du pays. Fait unique dans l'édition touristique, la section sur le trekking se distingue par une vision critique remarquablement réfléchiequi seclôt par un conseil qu'aucun autre éditeur touristique n'a jamais osé imprimer:
[...] many people feel awkward walking through bill-tribe villages and playing the role of voyeur. ?...] trekkers introduce many cultural items and ideas from tbe outside world that way erode tribal customs, to varying degres. If you bave any qualms about interrupting the traditional patterns of hife in bill- tribe areas, you probably should not 
go trekking. (Cumnings, 1990: 205)

En comparaison des ouvrages déjà cités, le visiteur bascule brutalement ici de l'état de spectateur bien intentionné, d'invité presque, à celui beaucoup moins confortant de facteur de dégradation culturelle. La frontière estainsi redessinée entre Soi etl'Autre, soudain quasi-hermétique, interdisant désormais la poursuite tranquille du rêve jovialiste, et amputant le trekking d'une de ses deux dimensions essentielles. Voix discordante dans le concert harmonieux des vendeurs de tribus, Lonely Plamet tient lieu d'exception qui, probablement, confirme la règle. Il semble raisonnable de penser que ses éditeurs n'ont pas d'intérêts financiers dans l'industrie du trekking en Thailande.

\section{De certaines récurrences}

Il paraît done n'y avoir, tant dans la littérature touristique que dans la production scientifique en sciences sociales, qu'un nombre très réduit de tentatives sérieuses de définir le terme trekking et, généralement, le sens du mot est considéré comme allant de soi par les auteurs. Dans les grandes lignes, si l'on tente un bilan, deux applications ressortent comme étant indissociablement liées au trekking. La dimension topographique d'abord. Si l'on marche dans les vallées himalayennes, si l'on aborde la jungle de Kalimantan, ou si l'on bivouaque dans quelque oasis du désert yéménite, on fait un trekking. Mais on fait une randonnée pédotre au Québec, à la rigueur on explore la toundra canadienne, ou on escalade un col des Rocheuses. Le terme trekking ne semble jamais s'appliquer aux systèmes urbains, ni même aux régions non-urbanisées de pays industrialises, fussent-elles pourvues des plus hautes montagnes ou recouvertes de la forêt la plus dense. Et il y a ensuite la dimension humaine, initiatique presque, porteuse de promesses de rencontre avec l'Autre et, à travers lui, avec le Soi individuel et le Nous universel. Il existe des sociétés primitives, intactes, vraies, preuves vivantes de la pureté révolue de notre propre existence. C'est un discours essentiellement rousseauiste prêchant la fuite - temporaire - de notre monde construit et cultivé, par l'immersion dans un bain de Nature nonsouillée. Le jeu manichéen sur la dichotomie Nature-Culture paraît être un ressort essentiel de la notion de trekking telle qu'elle est publicisée.
On pourrait difficilement vendre sous le nom de trekking une excursion qui n'incluraitpas explicitement la rencontrehumaine; ce serait alors une expédition, un rallye, etc., mais pas un trekking. Alors qu'il m'apparaît plausibleque la partie physique du trekking, loin d'être nécessairement la poursuite de l'exploit, ne soit en fin de compte que la matérialisation nécessaire du passage entre l'état de Culture et l'état de Nature. Il est possiblequ'une hiérarchie non-dite existe entre les deux dimensions essentielles de l'activité.

En observant surtout les sociétés occiden= tales, l'anthropologie a remarqué depuis un bon moment l'importance de l'alternance entre temps sacré et temps profane (Leach, 1961 par exemple). Boorstin(1962) et Turner \& Turner(1978) ont identifié en leur temps les ressorts de la motivation à la fuiteritualiséeetàla quête. Nelson Graburn (1977) a habilement appliqué ce constat au rôle similaire que joue le tourisme dans nos sociétés industrialisées. L'imagerie associée à l'inconnu, au départ, à la découverte, et bien sûr au retour, puisque tourisme n'est pas migration, révèle un cycle de quête de soi oủ le temps sacralisé- levoyage régénérateur = al terne immuablement avec le temps vulgaire - le quotidien - l'un fécondant l'autre et lui donnant un sens. Pèlerinage sans Dieu, retour aux sources, éternelle recherche de l'authenticité perdue, le trekking comme forme particulière de cette quête correspond bien à ce découpage de notre vie sociale. Mais le trekking est en même temps phus que le voyage, puisqu'il se place le plus souvent comme une parenthèse à l'intérieur même du voyage, entre les hôtels et les luxes des capitales à l'arrivée, et les plages ensoleillées en guise de récompense avant le retour. Dans les brochures publicitaires, un trekking faisant partie des multiples activités d'un itinéraire organisé n'est jamais présenté comme une activité parmi d'autres: c'est le coeur pal pitant du voyage, le moment fort, la référence dans le temps marquant l'avant et l'après. Durant un camaval, ce serait la transe.

Le fait est qu'il doit demeurer ainsi pour que le plus grand nombre puisse y projeter ses espoirs et ses fantasmes, et faire tourner la machine; c'est une simple question de mise en marché. L'important, au fond, c'est que le concept soit suffisamment opérationnel pour que l'industrie de l'aventure parvienne à l'utiliser sans mesure, à lui faire dire à peu près n'importe quoi, à en faire une expérience de polysémie appliquée dont la popularité sans démenti est sans doute la meilleure preuve de l'adéquacité à la réalité. À une certaine réalitế, à tout le moins.

\section{NOTES ET RÉFÉRENCES}

(1) La réflesion sur laquelle se fonde co texte se nourrit tant a l'bcriture recente d'une thabe de doctorat sur le theme du changement social et du tourisme dans un village d'ethnie Hmong du Nord thailandais, qu'a une expérience passece comme guide de trekking en Himalaya et au Mexique. Les testes scientifiques traitant spdofiquement du trekking etant a toutes fins pratiques inexistants. ce sera surtout ma connaissance directe du phenomene tant par la pratique qu'au moyen de I'examen scientifique, qui m'autonisera, i' aime a le croire, a etablir quelques observations.

(2) Le concept d'authenticite - en particulier ba notion de staged authenticity a fait couler beaucoup d'encre depuis vingt ans dans la recherche sociale sur le tourisme, en particulier depuis les cumrages marquants de MacCannell (1976) et de Tumer \& marquants de
Turner (1978).

ARMINGTON, S., Trekking in the Himalayas, Hawthorn (Australie), Lonely Planet Publications, 1982.

BAFFIE, J. Voyage a travers les guides: les guides de voyages de langue francaise sur la Thadande, Inter-Mondes, wol. 1, no 1. 1989, pp. 103-165.

BOORSTIN, $J$, the Image, Or What Happened to the American Dream, New York, Atheneum, 1962. CLUB AVENTURE, Repertoire 1991-1992. Montróal, Club de voyages Aventure inc. 1991, 51 p.

CLUa AVENTURE, Partons ensemble au coeur du monde, Monireal, Club Aventure Voyages, 1987. $136 \mathrm{p}$.

COHEN, E., Primitive and remote: Hill Tribe Trekking in Thailand, Annals of tourism Research, wol. 16 , no 1,1989 , pp. 30-61,

CUMMINGS, J. Thailand. A Traval Survival Kit Hawthorn (Australie). Lonely Planet Publications, 1990.

DAVIES J.R and T Wu A trakker's Guide to: the Hill Tribes of Northern Thailand, Salisbury/England), Footloose Books, 1990, $147 \mathrm{p}$.

EXPEDITIONS MONDE, Expeditions Monde 1992. 1993, Montréal, 1992, $23 \mathrm{p}$.

GRABURN, N.H., towism: the Sacred journey, in V.H. Smith (dir.). Hosts and Guests, the Anthropology of tourism. Oxford, Blackwell, 1977, pp. 17-31.

LE GUIDE DU ROUTARD, Le guide du routard 1992 . 1993. Thailande, Birmanis, Hong Konq. Macao 1993. Thailande, Birm
Paris, Hachette, 1992 .

LEACH, $E_{,}$Rethinking Anthropology, Lendes, Athlone Press, University of London, 1961.

MACCANNELL, D. the Tourist: A New Theory of Leisure Class, New York.

SMITH, V., introduction, in VH, Smith (dir). Hosts and Guests: the Anthropolegy of tourism, Philadelphia, University of Pennsylvania Press, 1977, Dp, $1-17$

TURNER, $V$, and $E$. TURNER, Image and Pilgrimage in Christian Culture. Anthropological Perspectives, Oxford, Blackwell, 1978 